\title{
Inflammatory Cytokinesinduce EMT in Mesenteric Mesothelial Cells, and Transdifferentiate Them into Macrophages
}

\author{
Anna L. Kiss
}

\begin{abstract}
Monocytes and macrophages are professional phagocytic cells and are the major differentiated mediators of immune responses. These cells are widely distributed in many tissues and organs. Under normal (steady state) conditions, large number of these phagocytic cells (called resident macrophages) reside in the peritoneal cavity and in the "milky spots" of the mesentery. As a result of inflammatory stimulus (i.e. intraperitoneal Freund's adjuvant injection) these resident peritoneal macrophages become activated, and their number and endocytic activity is significantly increase. The origin and plasticity of these chronic, heterogeneous inflammatory macrophages are not entirely known. Tissue-resident macrophages as well as infiltrating monocyte-derived macrophages play a distinct role in the progression of inflammation, but the large number of these inflammatory macrophages suggests that cells originating from other, non-hematopoietic sources can also contribute to this subset of macrophages. Our morphological and biochemical results provided evidence that under the effect of inflammatory cytokines mesenteric mesothelial cells undergo epithelial-mesenchymal transition (EMT) and transdifferentiate into macrophages, thus these transformed mesenteric mesothelial cells can contribute to the large increase of peritoneal macrophages during inflammation. In this paper, we summarize our morphological and biochemical data supporting this transition and we describe how cavolae and caveolar endocytosis can regulate both epithelial-mesenchymal transition (EMT) and mesenchymal-epithelial transition (MET).
\end{abstract}

Index Terms - Inflammation, inflammatory cytokines, mesenteric mesothelial cells, epithelial-mesenchymal transition, mesenchymal-epithelial transition, caveolae, caveolar endocytosis.

\section{INTRODUCTION}

This review summarizes our data proving that inflammatory stimuli induce epithelial-mesenchymal transition in mesenteric mesothelial cells. During this process, the simple squamous epithelial cells undergo a series of morphological and biochemical changes, lose their epithelial characteristics and become mesenchymal cells. The transforming growth factor $\beta$ (TGF $\beta)$ and granulocyte-macrophage colony stimulating factor (GM-CSF) play important role in regulation of this process. After the peak time of inflammation the mesothelial cells start to regenerate, they regain their original epithelial phenotype by a process called mesenchymal-epithelial transition (MET). Autophagy, by removing the unnecessary cellular organelles,

Anna L. Kiss, Department of Anatomy, Histology and Embryology, Semmelweis University Budapest, Hungary is the key process to re-establish their epithelial morphology. Our morphological and biochemical results clearly show that during inflammation induced EMT besidesof gaining mesenchymal characteristics, mesenteric mesothelial cell transdifferentiate into macrophages contributing the increased amount of macrophages appearing into the peritoneal cavity. We also prove that both EMT, MET and mesothelial cell-macrophage transdifferentiation (MMT) are initiated and regulated by inflammatory cytokines. Caveolae and caveolar endocytosis seem to be one of the important checkpoint in the regulation the signalling during both EMT, MET and MMT.

\section{INFLAMMATION INDUCES EPITHELIAL-MESENCHYMAL TRANSITION (EMT) IN MESENTERIC MESOTHELIAL CELLS}

The process of epithelial-mesenchymal transition [1,2] is an important, reversible biological process, during which polarized epithelial cells undergo a complex proteomic remodelling, several biochemical and morphological changes andassume mesenchymal characteristics [3]. In the course of this transdifferentiation the polygonal-shaped epithelial cellsbecome elongated, spindle-shaped, apolar, and highly mobile cells. The cells lose their intercellular connections(with E-cadherin and B-catenin down-regulation [4,5]), their basement membrane is degraded [6], and their cytoskeleton becomes rearranged. Besides expressing mesenchymal markers ( $\mathrm{N}$-cadherin, vimentin, $\alpha$-smooth muscle actin), epithelial cells acquire the ability to produce extracellular matrix components, inflammatory cytokines, fibrogenic and angiogenic factors [7]. Three types of EMT have been distinguished so far: a.) EMT occurring during embryogenesis (type I); b.) EMT associated with inflammation, wound healing, tissue regeneration and organ fibrosis (type II) and c.) tumorigenesis (type III) [8].

EMT is triggered by extracellular signals including various cytokines, growth factors, and extracellular matrix components. Transforming growth factor $\beta$ (TGF $\beta$ ) family members are the most important regulators in EMT [9]. As an important morphogenic factor, TGF- $\beta$ initiates and maintains EMT via different signaling routes[10]. The TGF $\beta$ signalling pathways are generally divided into Smad-dependent and Smad-independent processes [11]. When TGF $\beta$ binds to its primary serine/threonine kinase receptor (type II), it induces the formation of a heterotetrameric receptor complex. Type II receptor then trans-phosphorylates (activates) the signalling receptor (type I) that can initiate $\mathrm{Smad} 2 / 3$ signalling pathway.

We have previously demonstrated [12] that intraperitoneal 
injection of Freund's adjuvant inducedmassive inflammation of the mesenterium with marked changes in the morphology of the mesenteric mesothelial cell.Flat squamous epithelial cells became rounded or cuboidal in shape, and the volume of the cells as well as the number of the cell organelles were significantly increased. The cells have lost both their apical-basolateral polarity and cell-cell interactions,and many of them detached from the basement membrane. The epithelial cell type-specific intermediate filament protein, cytokeratin has gradually disappeared from the cytoplasm of the mesothelial cells with a parallel increase of the mesenchymal cell-specific vimentin expression.The inflammation resulted in an extensive cell migration, structural reorganization, and loss of cell continuity [12].

Freund's adjuvant treatment also induced a significant amount of TGF- $\beta$ production in mesothelial cells that was secreted into the peritoneal cavity. The level of TGF- $\beta$ highly correlated with the kinetics of the inflammation, and transforming mesothelial cells also expressed TGF $\beta$ RII[13]. These morphological and biochemical data show that under inflammatory stimuli mesenteric mesothelial cells undergo EMT, that is regulated by TGF- $\beta$ signalling.

\section{DURING RECOVERY, MESOTHELIAL CELLS UNDERGO MESENCHYMAL-EPITHELIAL TRANSITION MET AND AUTOPHAGY IS THE KEY PROCESS INTHIS REGENERATION}

After inflammation reaching its peak, a recovery process began: the mesothelial cells gradually regained their simple squamous epithelial morphology and cellular organization (a process called mesenchymal-epithelial transition, MET). The cells,being still cuboidal in shape, became arranged in a single layer on the surface of the mesentery, the volume of the cytoplasm gradually decreased, and finally only few intracellular organelles were present in the cytoplasm. Since no cell division could be detected that could have provided new mesothelial cells, we assumed that the regeneration of the mesothelium occurred by remodelling of the transformed mesothelial cells. In tissue remodelling,autophagy is known to play a pivotal role, by which the cells can control the number and turnover of cell organelles [14-17]. Recent studies show that autophagycan play an essential role in inflammatory processes as well[18,19]. At the start and subsequent development of regeneration a progressive autophagosome and autolysosome formation were found in our system [20]. Simultaneously with the morphological changes, the expression of various factors directly or indirectly regulating autophagy has also changed. At the early time of inflammation the Beclin-1 level prominently increased, was high at the peak of inflammation, and by the time of regeneration it completely disappeared.Although a basal Beclin-1level is necessary for the cell survival, it is not capable to trigger autophagy [21]. A major regulator of the autophagic processis mTOR[22] that integrates intra- and extracellular signals, and serves a regulatory role in cell growth, proliferation, metabolism and survival.Inhibition of mTOR increases autophagy, whereas its activation (by phosphorylation) reduces or inhibits the process [23]. mTOR is the downstream effector of PI3K-Akt system. Various cytokines and extracellular stimulican activate the serine/threonine protein kinase Akt or PKB (protein kinase B, a downstream effector of PI3K)as well as mTOR [22,25]. TGF- $\beta$,besides being the major regulator of EMT, can also activate the PI3K-Akt-mTOR pathway indirectly [26,27]. Since in our system TGF- $\beta$ is secreted into the peritoneal cavity during inflammation $[13,28]$, we assume that TGF- $\beta$ not only stimulates the EMT (through its canonical pathway), but it also arrests the autophagy by stimulating the phosphorylation of Akt-mTOR.When the inflammation progressed and the autophagy was arrested we found high p-Akt and p-mTOR levels. Following the peak time of inflammationboth p-Akt and p-mTOR levels diminished and the number of autophagic vacuoles increased significantly, indicating that simultaneously with the inactivationof regulatory molecules the autophagy could be accelerated [20]. These results show thatduring inflammation TGF- $\beta$ has a dual effect: through its plasma membrane receptor, it stimulates EMT (canonic pathway) andby stimulating PI3K-Akt pathway, indirectly inhibits autophagy.

Recently, estrogen receptor alpha (ER- $\alpha$ ) has been suggested as another player in the molecular regulation of EMT and MET [29-31]. For long, estrogen receptors (ER- $\alpha$, ER- $\beta$ ) have been considered exclusively as transcription factors acting inside the nucleus. It has been proved, however, that a small percentage (5-10\%) of ERs reside in the cell membrane and can elicit both genomic and non-genomic responses by activating multiple protein kinase cascades that include MAPK, protein kinase C, Src kinase and PI3K [32-36]. ER- $\alpha$, and TGF- $\beta$ have opposing roles in cell proliferation and differentiation of epithelial cells. Their regulatory pathways intersect and ER- $\alpha$ blocks the TGF- $\beta$ pathway at different cellular levels both inside the nucleus and in the cytoplasm and plasma membrane [35-38]. It is known that estrogen can activate the ERK pathway $[37,38]$ and this non-canonical MEK-ERK signalling regulates mTOR activation, thus controlling the intensity of autophagy [39]. Our morphological (confocal and immuno-EM) and biochemical (qRT-PCR) results showed that ER- $\alpha$ was expessed in both treated and in control mesothelial cells. Balogh et al [13]showed that the extragonadal estrogen, activating the non-canonical MAPK pathway through its receptor (ER $\alpha)$, could induce autophagy in our system as well. All these morphological and biochemical data provide evidence that autophagy is deeply involved in the re-establishment of the original mesothelial phenotype (MET) in our system by removing unnecessary cell organelles.

\section{ESSENTIAL ROLE OF CAVEOLAE AND CAVEOLAR ENDOCYTOSIS IN EMT AND MET}

Mesothelial cells exhibit numerous caveolae both at their apical and basal plasma membranes [40]. Caveolae are omega or flask shaped plasma membrane invaginations, highly hydrophobic membrane domains, considered as a specialized form of lipid rafts $[41,42]$. The major protein of caveolae is caveolin-1 $[43,44]$, which drives the formation of caveolae, anchors them to the actin cytoskeleton, modulates cell interactions with the extracellular matrix, regulates signalling molecules and cholesterol transport [45]. Since caveolin-1 - through its scaffolding domain - can bind many molecules implicated in signalling events, caveolae are thought to be important signalling organelles [43]. Recently it 
World Journal of Research and Review (WJRR) ISSN:2455-3956, Volume-7, Issue-2, August 2018 Pages 32-37

has been accepted that caveolae are also involved in endocytic processes [44]. Signalling and internalization however are strongly related to each other. The receptor internalization is required for the initiation of downstream signalling [46]. In addition to promoting the signalling process by transporting these molecules to the signalling partners in the early endosomes,the receptor-ligand internalization regulates the number of receptors present in the cell membrane. The two pathways from the early endosomes lead to recycling endosomes and multivesicular bodies/late endosomes. From the recycling endosomes, the receptors can return to the plasma membrane and take part in another cycle, thus the signalling can continue until receptors recycle back to the cell surface. As the alternative pathway, multivesicular bodies/late endosomes fuse with lysosomes and the receptors are degraded. Thus, receptor internalization regulates the signalling, receptor turnover, the magnitude and duration of the events. In the regulation of EMT clathrin-mediated as well as caveolar endocytosis (internalization via caveolae) are involved [47]. Internalization via clathrin-coated vesicles promotes the signalling, while the caveola-mediated endocytosis plays important role in termination of the events. During inflammation-induced EMT of mesenteric mesothelial cells both TGF $\beta R I$ and TGF $\beta$ RII are rapidly internalized upon ligand binding and theinternalized receptor-ligand complexesare targeted into early endosomes[28]. These compartments promote the signalling indicating their pivotal role not only in the sorting of internalized cargo proteins but in defining the activity of signalling events as well[48]. Endocytosis via caveolae directs the receptor-ligand complex first to early endosomes then to late endosomes/lysosomes or proteasomes, where they are degraded [49,50] resulting indownregulation of the receptors on the cell surface. In our system as the inflammation progressed, the TGFßRII appeared in caveolin-1 positive intracellular structures that was accompanied by the appearance of negative regulatory protein, Smad7 (an ubiquitin ligase) in caveolae[28]. Our fine structural, morphometric and immunoblot analysis proved that multivesicular body formation was significantly increased during inflammation and both TGFßRII and caveolin-1 were strongly associated with these compartments.By the termination of inflammation, caveolin-1 was found to be associated with the late endosomal marker,Rab7 and entirely degraded in these compartments [28]. The most debated and controversial itemconcerningthe regulatory role of early endosomes and caveolae in TGF- $\beta$ pathway is whether caveolae directly reach late endosomal compartments (multivesicular bodies/late endosomes) or only with the fusion of early endosomal membranes. Our light and electron microscopical results have shown that caveolae, internalized upon inflammatory stimuli, most likely meet first the early endosomes, then reach the multivesicular body compartment, thus early endosomes are also functioning as the key compartments in the termination of TGF- $\beta$ signalling.As a conclusion, we assume that these findings provide persuading evidence how caveolin-1 downregulation together with caveolar internalization of TGF $\beta$ receptors turns off the TGF $\beta$ signalling pathway and blocks EMT[28]. Caveolar endocytosis ofTGF $\beta$ receptors can also regulate the signalling events of autophagy. As discussed above, TGF- $\beta$ can also activate indirectly the PI3K-Akt-mTOR pathway [26,27],stimulating the phosphorylation of Akt-mTOR. Stimulation (phosphorylation) of mTOR reduces, inhibits [23], while its inhibition stimulatesautophagy. When theTGF $\beta$ receptor is internalized, the ligand cannot transmit the stimulus to the downstream molecules, and EMT is blocked. In our system, it also means that by the receptor internalizationthe inhibitory effect of the downstream signalling is stopped, mTOR can initiate autophagy, and the recovery (MET) can start[20].From these data, we conclude that the receptor internalization can be one of the most important steps in allowing autophagy through inactivation of PI3K-Akt-mTOR pathway as well.

Summarizing: caveolae, the omega-shaped plasma membrane invaginations, have multiple functions in signalling events. They provide assembly platforms for various signalling molecules that can be regulated by the interaction with caveolin-1. The caveolar internalization of receptor-ligand complexes controls the number of the specific receptors present on the cell surface, and determines the capability of the cell to respond to extracellular stimuli. Therefore caveolar internalization of cytokine (TGF $\beta$, BMPs, etc.) receptors regulates the intensity of both EMT, and MET; the balance between EMT and MET highly depends on the presence of caveolin-1/caveolae on the plasma membrane [7,51].Consequently, caveolin-1 is likely to be the major checkpoint in epithelial-mesenchymal and mesenchymal-epithelial transitions [7].

\section{MESOTHELIAL CELL-MACROPHAGE TRANSITION (MMT) DURING INFLAMMATION INDUCED EMT}

During inflammation-induced EMT mesenteric mesothelial cells start to express macrophage markers (OX43, ED1) as well [12,52], suggesting that they might be transformed into macrophage-like cells [53]. Our previous data showed that under inflammatory stimuli the number of peritoneal macrophages dramatically increased [54]. The origin of these chronic, heterogeneous inflammatory macrophages is not entirely known. There are data indicating that tissue-resident macrophages as well as infiltrating monocyte-derived macrophages play a distinct role in the progression of inflammation [55-58]. The large number of the inflammatory macrophages suggests, however, that blood-derived monocytes, and activated resident macrophages should not be enough to provide such a large amount of macrophages, and cells originating from other, non-hematopoietic sources should also contribute to this subset of macrophages. One candidate of these non-hematopoietic cells are the mesothelial cells that are present all over the peritoneal cavity, covering the internal organs of the abdomen. They express nestin [12], a well-known and characteristic marker of multi-lineage progenitor cells, the presence of which indicates multipotentiality and regenerative capacity [59]. Nestin expression in mesothelial cells clearly shows that they are not terminally differentiated cells;instead, they are multipotent ,young" cells with high regenerative capacity and able to differentiate into many types of cells [12].

The granulocyte-macrophage colony-stimulating factor (GM-CSF) - a member of the hematopoietic cytokine family promotes the survival and activation of granulocytes, 
macrophages and dendritic cell differentiation in vivo, and it also stimulates proliferation of several non-hematopoietic cell types (osteoblasts, smooth muscle, endothelial and epithelial cells) [60]. During inflammation, mesenteric mesothelial cells synthesize GM-CSF and secrete it into the peritoneal cavity [53]. If treated with GM-CSF, mesothelial cells started to express macrophage markers [53], showing that GM-CSF initiated the mesothelial cell-macrophage transition (MMT).Upon inflammatory stimulus as well as GM-CSF-treatment, the phagocytic activity ofmesothelial cells significantly increased,theyefficiently phagocytosed both India ink and fluorescent bioparticles [62]. Besides being professional phagocytic cells, macrophages are the major producers of TNF $\alpha$, which is the main regulator of inflammatory cytokine production [61]. During inflammation, mesothelial cells expressed an increasing amount of TNF $\alpha$, and EGR1[62]. EGR1(early growth response 1) transcriptional factor is a member of early-immediate growth response gene family coupling the acute perturbation of the extracellular milieu to short-lived changes in target gene expression[63]. As a unique transcription factor, EGR1 affects only cells of the monocyte/macrophage lineage, not other haematopoietic cells [54,64-67]. We could detect EGR1 inthe nuclei of the mesothelial cells indicating that inflammation resulted in not only the expression but also the nuclear translocation of this transcription factor. EGR1 activity and its nuclear translocation depend on ERK activation [68], and caveolin-1 is known to regulate ERK1/2 phosphorylation, positively affecting the monocyte to macrophage differentiation [68]. In our system, we found an increased ERK1/2 phosphorylation at a maximum level on day 3 of inflammation and caveolin-1 expression also changed during inflammation indicating that mesothelial cell-macrophage transdifferentiation (MMT) is regulated by caveolin-1/ERK1/2/EGR1 signaling pathway[62].

Another characteristic feature of macrophages is that under inflammatory stimuli they express and secrete pro-inflammatory cytokines such as TNF $\alpha$, IL-1, IL-6, IL-8, IL-12, as well as anti-inflammatory cytokines like IL-10, TGF $\beta$ [69]. Cytokines and chemokines are potent signalling molecules with low molecular weight, mediating intercellular communication. The primary function of cytokines is to regulate inflammation by paracrine, autocrine or endocrine mechanisms. Pro- and anti-inflammatory cytokines are mainly produced by macrophages and lymphocytes, but polymorphonuclear leukocytes (PMN), endothelial, epithelial cells, adipocytes and connective tissue cells are also able to synthesize them [69].

We found that mesenteric mesothelial cells also expressed both pro- and anti-inflammatory cytokines to regulate inflammation (unpublished data). In control mesenteric mesothelial cells we could detect the anti-inflammatory cytokine, IL-10, which is known to suppress macrophage activation, to improve cell survival and to reduce the levels of inflammatory cytokines [70]. As the inflammation progressed, mesothelial cells started to express the pro-inflammatory cytokine IL-6 (unpublished data), that regulates the expression of inflammatory genes [71], and simultaneously the IL-10 level have been decreased. At the peak of inflammation, IL-6 reached its maximum level, while IL-10 practically disappeared from the cells. As the regeneration started, IL-6 expression decreased and IL-10 level started to increase. From these data, we conclude that mesenteric mesothelial cells, developing from the embryonic splanchnopleura, preserve their multipotent mesenchymal character, and in response to special stimuli (inflammation, GM-CSF treatment) can transdifferentiate into macrophages. In addition to emigrating blood monocytes and resident macrophages, they could provide the third source of peritoneal macrophages during inflammation. The caveolin-1/ERK1/2/EGR1 signaling pathway regulates this mesothelial cell-macrophage transition.

It is well known that various signals, such as apoptotic cells, hormones, immune complexes and cytokines, can induce polarization of macrophages. Under the effect of diverse signals macrophages can become "classically activated"(M1) or "alternatively activated"(M2a, M2b, M2c) cells [72]. M1 macrophages secrete pro-inflammatory cytokines such as TNF and IL-6. Exposure or treatment of monocytes with IL-4 and IL-13 polarizes these cells toward M2a [73,74]. M2b macrophages are induced by a combination of LPS, immune complexes, apoptotic cells and IL-1Ra. They secrete both IL-10 and IL-6, while M2c macrophages are induced by a combination of IL-10, TGF $\beta$ and glucocorticoids, in turn they secrete IL-10 and TGF $\beta$. They also promote tissue regeneration $[73,74]$. Taking together all these data and our results, we think that control mesothelial cells have M2c macrophage character, and the inflammatory stimuli polarize them into M2b and M1 macrophages.

All these data and facts point out and underline that mesenteric mesothelial cells are not terminally differentiated cells instead they are multipotent plastic cells, they can be taken as a kind of "stem" cells. During inflammation, under the effect of inflammatory cytokines, because of their plasticity, mesenteric mesothelial cells are capable to transdifferentiate into macrophages, by a process called EMT. This transdifferentiation however is reversible; during this transition, they do not lose their mesothelial character: if the inflammatory stimulus is over, they can regain their mesothelial phenotype again.

\section{CONFLICT OF INTEREST}

The authors have declared that no competing interest exists.

\section{ETHICAL APPROVAL}

All applicable international, national, and/or institutional guidelines for the care and use of animals were followed.

\section{ACKNOWLEDGEMENT}

I would like to express my thankfulness to Professor Pál Röhlich for the precious comments and accurate language correction of the manuscript. Special thanks to Katalin Lőcsey and Nikolett Dóczi for their valuable technical help, and to my colleagues Sándor Katz, Petra Balogh and Viktória Zsiros for the experiments they have done.

\section{REFERENCES}

[1] E.D.Hay.Epithelia suspended in collagen gels can lose polarity and express cahracteristics of migrating mesenchymal cells. J. Cell. Biol. 95(1)(1982) 333-339. 
World Journal of Research and Review (WJRR)

ISSN:2455-3956, Volume-7, Issue-2, August 2018 Pages 32-37

[2] E.D.Hay.The mesenchymal cell, its role in the embryo, and the remarkable signaling mechanisms that create it. Dev. Dyn. 233 (2005) 706-720.

[3] R. Kalluri, R.A. Weinberg. The basic of epithelial-mesenchymal transition. J. Clin. Invest. 119 (2009)1420-1428.

[4] P. Savagner. Leaving the neighbourhood: Molecular mechanisms involved during epithelial-mesenchymal transition. Bioassay 23 (2001) 912-923.

[5] B. Boyer, A.M. Valles, N. Edme. Induction and regulation of epithelial-mesenchymal transitions. Biochem. Pharmacol. 60 (2000) 1091-1099.

[6] J. Yang, Y. Liu. Dissection of key events in tubular epithelial to myofibroblast transition and its implication in renal interstitial fibrosis. Am. J. Pathol. 159 (2001) 1465-1475.

[7] R. Strippoli, J. Louriro, V. Moreno, I. Benedicto, M.L.P. Lozano, O. Barreiro, T. Pellinen, S. Minguet, M. Foronda,M.T. Osteso, E. Calvo, J. Vázquez, M.L. Cabrera, M.Q.A. del Pozo, Caveolin deficiency induces a MEK-Erk1/2-Snail-1-dependent epithelial-mesenchymal transition and fibrosis during peritoneal dilysis EMBO Mol. Med. 7 (2015) 102-123.

[8] [8] M.J.Lee,S.Debhar, R.Kalluri, W.E.Thompson. The epithelial-mesenchymal transition: newinsights in signalling, development, and disease. J. Cell. Biol.172 (2006) 973-981.

[9] J. Massagué. TGF- $\beta$ signal transduction. Ann. Rev. Biochem. 67 (1998) 753-791.

[10] J. Zavadil, E.P. Böttinger. TGF- $\beta$ and epithelial-to-mesenchymal transitions. Oncogene24 (2005) 5764-5774.

[11] R. Derynck, Y.E. Zhang. Smad-dependent and Smad-independent pathways in TGF- $\beta$ family signalling. Nature 424 (2003) 577- 584.

[12] S. Katz, P. Balogh, A.L. Kiss. Mesothelial cells can detach from the mesentery and differentiate into macrophage-like cells. APMIS 119 (2011) 782-793.

[13] P. Balogh, A. Szabó, S. Katz, I. Likó, A. Patócs, A.L. Kiss. Estrogen receptor alpha is expressed in mesenteric mesothelial cells and is internalized in caveolae upon Freund's adjuvant treatment. PLoS One8(2013); doi: 10.1371/journal.pone.0079508.

[14] M.Gajewska,K.Zielniok, T.Motyl. Autophagy in Development and Remodelling of Mammary Gland, in: Autophagy - A Double-Edged Sword - Cell Surviv. or Death?, InTech, (2013) doi:10.5772/54558.

[15] N.Mizushima,M.Komatsu. Autophagy: Renovation of Cells and Tissues Cell 147 (2011) 728-741. doi:10.1016/j.cell.2011.10.026.

[16] S.Periyasamy-Thandavan, M.Jiang, P.Schoenlein, Z.Dong. Autophagy: molecular machinery, regulation, and implications for renal pathophysiology. Am. J. Physiol. Renal Physiol.297 (2009) 244-256. doi:10.1152/ajprenal.00033.2009.

[17] N.Mizushima. The pleiotropic role of autophagy: from protein metabolism to bactericide. Cell Death Differ. 12.(2005) 1535-1541. doi:10.1038/sj.cdd.4401728.

[18] A.J.S.Choi, S.W. Ryter. Autophagy in inflammatory diseases. Int. J. Cell Biol.(2011) 732-798

[19] B. Levin, N. Mizushima, H.W. Virgin. Autophagy in immunity and inflammation. Nature, 469 (2011) 323-335.

[20] V. Zsiros, K. Katz, N. Dóczi, A.L. Kiss. Autophagy is the key process in the re-establishment of the epitheloid phenotype during mesenchymal-epithelial transition (MET). Exp. Cell Res. 352 (2017) 382-392.
[21] R. Kang, H.J. Zeh, M.T. Lotze, D. Tang. The Beclin1 network regulates autophagy and apoptosis. Cell Death Differ. 18 (2011) 571-580.

[22] M. Laplante, D.M. Sabatini. MTOR signalling in glance. J. Cell Science 122 (2009) 3589-3594.

[23] P. Codogno, A.J. Meijer. Autophagy and signaling: their role in cell survival and cell death.Cell Death Differ. 12(2) (2005)1509-1518. doi:10.1038/sj.cdd.4401751.

[24] G. Song, G. Ouyang, S. Bao. The activation of Akt/PKB signalling pathway and cell survival. J. Cell. Mol. Med. 9 (2005) 59-71.

[25] J. Wang, M.W. Whiteman, H. Lian, G. Wang, A. Singh, D. Huang, T. Denmark. A non-canonical MEK/ERK signalling pathway regulates autophagy via regulating Beclin 1. J. Biol. Chem. 284 (2009) 21412-2124.

[26] S. Lamouille, R. Derynck. Cell size and invasion in TGF-beta-induced epithelial to mesenchymal transition is regulated by activation of the mTOR pathway. J Cell Biol. 178 (2007) 437-451.

[27] S. Lamouille, E. Connolly, J.W. Smyth, R.J. Akhurst, R. Derynck TGF-beta induced activation of mTOR complex 2 drives epithelial-mesenchymal transition and cell invasion. J. Cell Sci. 125 (2012) 1259-1273.

[28] P. Balogh, M. Magyar, A. Szabó, N. Müllner, I. Likó, A. Patócs, A.L. Kiss. The subcellular compartmentalization of TGF $\beta$ RII and the dynamics of endosomal formation during the signalling events: An in vivo study on rat mesothelial cells. Eur. J. Cell Biol. 94 (2015) 179-234.

[29] Y. Ye, Y. Xiao, W. Wang, K. Yeasley, J.X. Gao, et al. ERalpha signaling through slug regulates E-cadherin and EMT. Oncogene 29 (2010) 1451-1462.

[30] M.D. Planas-Silva, P.K. Waltz. Estrogen promotes reversible epithelial-to-mesenchymal-like transition and collective motility in MCF-7 breast cancer cells. J. Steroid Biochem. Mol. Biol. 104 (2007) $11-21$.

[31] I.K. Guttila, B.D. Adams, B.A.White. ER $\alpha$, microRNAs, and the epithelial-mesenchymal transition in breast cancer. Trends Endocrinol. Metab. 23 (2012) 73-82.

[32] M. Razandi, A. Pedram, G.L. Greene, E.R. Levin. Cell membrane and nuclear estrogen receptors derive from a single transcript: studies of ER $\alpha$ and ER $\beta$ expressed in CHO cells. Mol. Endocrinol. 13 (1999) 307-319.

[33] R. Pietras, C.M. Szego. Specific binding sites for oestrogen at the outer surfaces of isolated endometrial cells. Nature 265 (1977) 69-72.

[34] A. Migliaccio, M. Di Domenico, G. Castoria, A. de Falco, B Bontempo, et al. Tyrosine kinase/p21 ras/MAP-kinase pathway activation by estradiol-receptor complex in MCF-7 cells. EMBO J. 15 (1996) 1292-1300.

[35] R.X. Song, Z. Zhang, R.J. Santen. Estrogen rapid action via protein complex formation involving ERalpha and Src. Trends Endocrinol. Metab. 16 (2005) 347-353.

[36] R.X. Song, R.J. Santen. Membrane initiated estrogen signaling in breast cancer. Biol. Reprod. 75 (2006) 9-16.

[37] T. Simoncini, P. Mannella, L. Fornari, A. Caruso, G. Varone et al Genomic and non-genomic effects of estrogens on endothelial cells. Steroids 69 (2004) 537-542.

[38] E.R. Levin. Plasma membrane estrogen receptors. Trends Endocrinol. Metab. 20 (2009) 477-482.

[39] M.K. Lee, C. Pardoux, M.C. Hall, P.S. Lee, D. Warburton, et al. TGF- $\beta$ activates Erk MAP kinase signaling through direct phosphorylation of ShcA. EMBO J. 26 (2007) 3957-3967. 
[40] C.J. von Ruhland, L. Campbell, M. Gumbleton, B. Jasani, G. Newman, Immunolocalization of caveolin-1 in rat and human mesothelium J. Histochem. and Cytochem. 52 (2004) 1415-1425.

[41] D.A. Brown, E. London. Functions of lipid rafts in biological membranes. Ann. Rev. Cell Dev. Biol. 14 (1998) 111-1136.

[42] K. Simons, D.Toomre. Lipid rafts and signal transduction. Nat. Rev. Mol. Cell. Biol. 1 (2000) 31-39.

[43] M.P. Lisanti, P. Scherer, Z.L. Tang, M. Sargiacomo. Caveolae, caveolin and caveolin-rich membrane domains: a signalling hypothesis. Trends Cell Biol. 4 (1994) 231-235.

[44] J. Couet, S. Li, T. Okamoto, P. Scherer, M.P. Lisanti. Molecular and cellular biology of caveolae: paradoxes and plasticities. Trends Cardiovasc. Med. 7 (1997) 103-110.

[45] A. Navarro, B. Anand-Apte, M-O. Parat. A role of caveolae in cell migration. The FASEB J. 18 (2017) 1801-1811.

[46] [46] K.H. Wrighton, X. Lin, X-H. Feng. Phospho-control of TGF $\beta$ superfamily signalling. Cell Res. 19(1) (2008) 8-20.

[47] C. Le Roy, L. Wrana. Clathrin- and non-clathrin-mediated endocytosis regulation of cell signalling. Nat. Rev. Cell. Biol. 6 (2005) 112-1126.

[48] [48] S. Hayes, A. Chawla, S. Corvera. TGF $\beta$ receptor internalization into EEA1-enriched early endosomes: role in signaling to Smad2. J. Cell Biol. 158 (2002) 1239-1249.

[49] G.M. Di Guglielmo, C. Le Roy, A.F. Goodfellow, L. Wrana. Distinct endocytic pathways regulate TGF- $\beta$ receptor signalling and turnover. Nat. Cell Biol. 5 (2003) 410-421.

[50] T.Ebisawa, M.Fukuchi, G.Murakami, K.Chiba, T.Imamura, K Miyazono. Smurf1 interacts with transforming growth factor-beta type-I through Smad7 and induces receptor degradation. J. Biol. Chem. 276 (2001) 12477-12480.

[51] D.M. Kingsley. The TGF-beta superfamily: new members, new receptors, and new genetic tests of function in different organisms. Genes Dev. 8 (1994) 133-146.

[52] S. Katz, P. Balogh, N.Nagy, A.L. Kiss. Epithelial-to-mesenchyma transition induced by Freund's adjuvant treatment in rat mesothelia cells: a morphological and immunocytochemical study. Pathol Oncol Res 2012;18:641-649.

[53] [53] S.Katz, V.Zsiros, N.Dóczi, A.Szabó, A.Biczó, A.L.Kiss GM-CSF and GM-CSF receptor have regulatory role in transforming rat mesenteric mesothelial cells into macrophage-like cells. Inflamm. Res.65 (2016) 827-836

[54] [54] A.L.Kiss,A. Kittel.Early endocytotic steps in elicited macrophages: omega-shaped plasma membrane vesicles at their cell surface. Cell. Biol. Int. 9 (1995) 527-538.

[55] [55] L.A.Ginsel, L.P. Rijfkogel,W.T.Deams. A dual origin of macrophages? Review and hypothesis, Macrophage Biology (1985) 621-649.

[56] [56] J.M.De Bakker, A.W.de Wit, H.Woelders, L.A.Ginsel, W.T.Deams. On the origin of peritoneal resident macrophages. II. Recovery of the resident macrophage population in the peritoneal cavity and milky spots after peritoneal cell depletion. J. Submicrosc. Cytol. 7 (1985) 141-151.

[57] J.M.Papadimitriou, R.B.Ashman. Macrophages: current view on their differentiation, structure and function. Ultrastruct. Pathol. 13 (1989) 343-372.

[58] F. Geissmann,M.G.Manz, S.Jung, M.H.Sieweke, M.Merad, K.Ley. Development of monocytes, macrophages and dendritic cells. Science 327 (2010) 656-661.

[59] C.Wiese, A.Rolletschek, G.Kania, P.Blyszczuk, K.V.Tarasova, R.P.Wersto, et al. Nestin expressiona property of multi-lineage progenitor cells? Cell. Mol. Life Sci. 61 (2004) 2510-2522.
[60] J.E.J.Rasko, M.M.Grough. Granulocyte macrophage-colony stimulating factor. In: Thomson, A.W. editor Cytokine handbook 2 ed. New York Academic Press(1994) 342-369.

[61] N. Parameswaran, S. Patial. Tumor necrosis factor- $\alpha$ signaling in macrophages. Crit. Rev. Eukaryot. Gene Expr. 20(2) (2010) 87-103.

[62] S. Katz, V. Zsiros, N. Dóczi, A.L. Kiss. Inflammation-induced epithelial-to-mesenchymal transition and GM-CSF treatmen stimulate mesenteric mesothelial cells to transdifferentiate into macrophages. Inflammation (2018) DOI $10.1007 / \mathrm{s} 10753-018-0825-4$

[63] V. Baron, E.D. Adamson, A. Calogero, G.F.Ragona, D. Mercola The transcription factor Egr1 is a direct regulator of multiple tumor suppressors including TGFbeta1, Pten, p53 and fibronectin. Cancer Gene Ther. 13 (2006) 115-124

[64] P.Laslo, C.J.Spooner,A.Warmflash, D.W.Laneki,H.J.Lee, R.Sciammas, B.N.Gantner, A.R.Dinner, H.Singh. Multilineage transcriptional priming and determination of alternate hematopoietic cell fates. Cell 126 (2006) 755-766

[65] K. Krishnaraju, H.Q. Nguyen, D.A. Liebermann, B. Hoffman.The zinc finger transcription factor EGR1 potentiates macrophage differentiation of hemopoietic cells. Mol. Cell Biol. 15 (1995) 5499-5507.

[66] K. Krishnaraju, B. Hoffman, D.A. Liebermann. The zinc finger transcription factor EGR-1 activates macrophage differentiation in M1 myeloblastic leukemia cells. Blood 92 (1998) 1957-1966.

[67] K. Krishnaraju, B. Hoffman, D.A. Liebermann. Early growth response gene 1 stimulates development of granulocyte and erythroid lineages. Blood 97 (2001) 1298-1305.

[68] Y.Fu, X-L.Moore, M.K.S.Lee, M.A. Fernandez-Rojo, M-O.Parat R.G.Parton, P.J.Meikle, D.Sviridov, J.P.F. Chin-Dustin. Caveolin-1 plays a critical role in differentiation of monocytes into macrophages. Arterioscer Thromb Vas Biol 32(2012) 117-125.

[69] G.A. Duque, A. Descoteaux. Macrophage cytokines: involvement in immunity and infectious diseases. Frontiers in Immunology 5 (2014) $1-12$

[70] F.N. Varzaneh,B. Keller, S. Unger, A. Aghamohammadi, K. Warnatz, N. Rezaei. Cytokines in common variable immunodeficiency as signs of immune dysregulation and potential therapeutic targets. J. Clin. Immunol. 34(5) (2014) 524-543.

[71] T. Taga, T. Kishimoto., Gp130 and the interleukin-6 family of cytokines. Ann. Rev. Immunol. 15 (1997) 797-819.

[72] E.S. Vanden, S. Goriely, D. De Wit, F. Willems, M. Goldman. IL-23 up-regulates IL-10 and induces IL-17 synthesis by polyclonally activated naive $T$ cells in human. Eur. J. Immunol. 35(2) (2005) 469-475

[73] S.K. Biswas, S. Mantovani. Macrophage plasticity and interaction with lymphocyte subsets: cancer as a paradigm. Nat. Immunol 11(10) (2010) 889-896.

[74] U.M. Gundra, N.M. Girgis, D. Ruckerl, S. Jenkins, L.N.Ward, Z.D Kurtz, et al. Alternatively activated macrophages derived from monocytes and tissue macrophages are phenotypically and functionally distinct. Blood 123 (2014) 110-122. 